\title{
MINIMUM ENERGY TRAJECTORIES FOR SUBWAY SYSTEMS
}

\author{
MANUEL A. DUARTE* AND PATRICIA X. SOTOMAYOR \\ Department of Electrical Engineering, University of Chile. Av. Tupper 2007, Casilla 412-3, Santiago, Chile
}

\begin{abstract}
SUMMARY
The problem of determining the tunnel trajectory together with an optimal control policy of a train in subway systems is addressed in this paper. The cost function chosen in this study is the total energy consumed by a train in a round trip. Several constraints such as maximum velocity, maximum slopes, maximum acceleration, maximum electrical force and so on, as well as a relatively simple model of the train are considered. The solution is obtained by using the Gradient-Restoration method developed by Angelo Miele et al. An application considering the kind of trains existing in the Santiago subway is presented and the results are compared with the existing tunnel profile from an energy consumption viewpoint. Important energy savings are obtained due to differences in proposed and existing tunnels, assuming that in both cases the operation policy is the same (optimal for a given tunnel). Copyright (C) 1999 John Wiley \& Sons, Ltd.

KEY WORDS optimal control; control applications; computer simulation; electric locomotive;

iterative methods; numerical methods; railways; transportation control; guided vehicles
\end{abstract}

\section{INTRODUCTION}

Energy consumption in underground systems is quite significant and represents an important part of the overall cost operation of electric railway systems. Thus, any attempt to improve operation efficiency will result in important energy savings. The latter can be accomplished in two different ways. Designing an optimal control policy (from an energy viewpoint) to operate trains under certain conditions and for a given tunnel trajectory will result in an energy saving. This type of solution is appropriate for existing underground systems which are not properly operated. Several results on this subject have been reported in the literature. ${ }^{1-8}$ All of them attempt to optimize the operation of existing systems, that is to say systems with a given track. Adaptive control techniques have also been explored to face this type of problem. ${ }^{9}$ There is another approach which consists in designing the tunnel trajectories simultaneously with generating an optimal operation policy for trains. This kind of design can only be applied to proposed systems since the cost of modifying actual tunnel trajectories could be prohibitive. To the knowledge of the authors, there are no theoretical results regarding this approach. Hoang ${ }^{10}$ and Duarte ${ }^{11}$ use an heuristic approach to determine suboptimal tunnel trajectories.

In this paper the problem of computing optimal tracks as well as drawing up a minimum energy policy for electric railways is addressed using numerical techniques rather than an heuristic approach. The problem can be considered as a double optimization with respect to control policy as well as train track.

\footnotetext{
* Correspondence to: M. A. Duarte, Department of Electrical Engineering, University of Chile, Av. Tupper 2007, Casilla 412-3, Santiago, Chile

CCC 0143-2087/99/060283-14\$17.50

Copyright (C) 1999 John Wiley \& Sons, Ltd. 
No electric regeneration is considered during braking periods so that optimization is performed from the starting point until the point at which the train starts braking.

A second-order, non-linear model is considered in the study together with several operational constraints. Particular values of parameters and special considerations regarding the type of rolling material and topology of Line No 1 (Escuela Militar-San Pablo) of the Santiago subway are used in the application shown in this paper.

\section{TRAIN MODELLING}

In this section, the equations describing the motion of the train are presented. The main simplificatory hypotheses are briefly stated to justify the rather simple train model utilized in this study. As a consequence, a non-linear, lumped-parameter dynamical model is obtained. Since the problem constraints treated in the next section are better expressed in term of the horizontal displacement ' $L$ ' rather than time ' $t$ ', we will state the model equations in terms of ' $L$ ' as independent variable, eventhough the resulting equations are more complex than those obtained using the time as independent variable.

In what follows, it has been assumed that tunnel trajectory lies on the equivalent vertical plane as shown in Figure 1. This simplification allows to analyse the problem in two rather than three dimensions. Thus, train position is completely defined at every instant of time by horizontal displacement (distance) $L(t)$ and vertical displacement (height) $h(t)$.

From physical considerations ${ }^{4,5,10,12}$ we can write the following equations describing the train movement:

$$
\begin{gathered}
\mathrm{d} v_{a} / \mathrm{dL}=1 / v_{a}\left\{\gamma_{a}-\left[k_{1}+k_{2} v_{a}^{2}\right]-k_{3} \sin \left(\theta_{a}\right)\right\} \\
\mathrm{d} t_{a} / \mathrm{d} L=1 / v_{a} \\
h_{a}(L)=\int_{0}^{L} \operatorname{tg}\left[\theta_{a}(L)\right] \mathrm{d} L, \quad h_{a}(0)=0 \\
\mathrm{~d} v_{b} / \mathrm{d} L=1 / v_{b}\left\{\gamma_{b}-\left[k_{1}+k_{2} v_{b}^{2}\right]-k_{3} \sin \left(\theta_{b}\right)\right\} \\
\mathrm{d} t_{b} / \mathrm{d} L=1 / v_{b} \\
h_{b}(L)=h_{\mathrm{BA}}+\int_{0}^{L} \operatorname{tg}\left[\theta_{b}(L)\right] \mathrm{d} L, \quad h_{b}(0)=h_{\mathrm{BA}}
\end{gathered}
$$

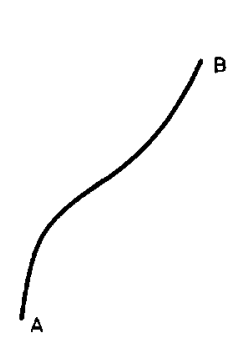

BOTTOM VIEW

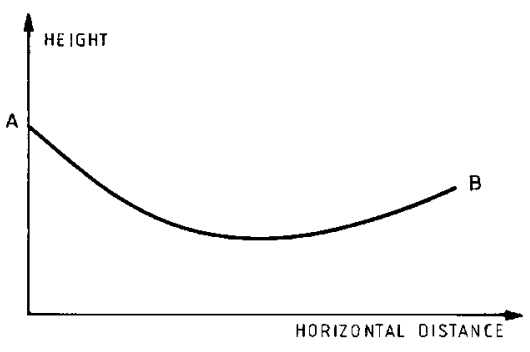

VERTICAL PROJECTION

Figure 1. Tunnel profile in bottom and equivalent vertical planes 
The subindex ' $a$ ' denotes the variable when the train is travelling from stations A to B, measured from station A, whereas the subindex ' $b$ ' denotes the variable when train travels from stations $B$ to A, measured from station B (see Figure 2). A distinction between these two sets of variables has to be made since our aim will be to minimize the round trip energy consumption (from stations $A$ to $\mathrm{B}$ and from stations $\mathrm{B}$ to $\mathrm{A}$ ) and therefore we have to consider both cases in a different way. The variables are defined as follows:

$h_{\mathrm{BA}} \quad$ level difference of station $\mathrm{B}$ with respect to station A

$h \quad$ vertical displacement of train (height) measured from station $\mathrm{A}$

$v \quad$ train velocity

$t \quad$ time elapsed

$\gamma \quad$ tractive force per unit of effective mass, delivered by the electric system

$\theta \quad$ slope angle of tunnel trajectory (track's slope)

$L \quad$ horizontal displacement of train (distance) from starting station

$k_{1} \quad$ characteristic constant to include friction effects between wheels and track, and other mechanical frictions

$k_{2} \quad$ characteristic constant to account for air resistance effects

$k_{3} \quad$ characteristic constant to include gravitational effects

Some of the model variables are shown in Figure 2 where train is represented by a mass ' $m$ ' at position $(L, h(L))$ at certain instant of time.

In the set of equations (1) amongst others, the following simplifications have been introduced.

(i) The train velocity is such that friction effects proportional to velocity are negligible.

(ii) The slope angle of track is small so that $\operatorname{tg}(\theta) \approx \theta, \sin (\theta) \approx \theta$ and $\cos (\theta) \approx 1$ is verified.

(iii) Train mass ' $M$ ' is assumed to be concentrated and the concept of effective mass ' $m$ ' rather than the actual mass of train is used ${ }^{4}$ to include inertial effects and train length. This effective mass is assumed to be constant.

(iv) Parameters $k_{1}, k_{2}$ and $k_{3}$ are assumed to be constant.

However the simple resulting model contains the main ingredients of real process and it is representative of the physical situation.

\section{PROBLEM CONSTRAINTS}

This section is devoted to constraint analyses. These constraints are present in the actual operation of any subway. The numerical values chosen in this work correspond to the case of Santiago subway.

The constraints to be taken in to account are listed below and a brief explanation is given in each case.

\subsection{Maximum velocity}

Since traction motors can deliver only a nominal electric power to the mechanical system, the maximum mechanical force reachable from electric system is limited. This fact, together with the physical consideration that train velocity cannot be negative, gives rise to the constraint

$$
0<v(L) \leqslant V_{\mathrm{M}}(L), \quad L \in\left[0, L_{\mathrm{f}}\right]
$$




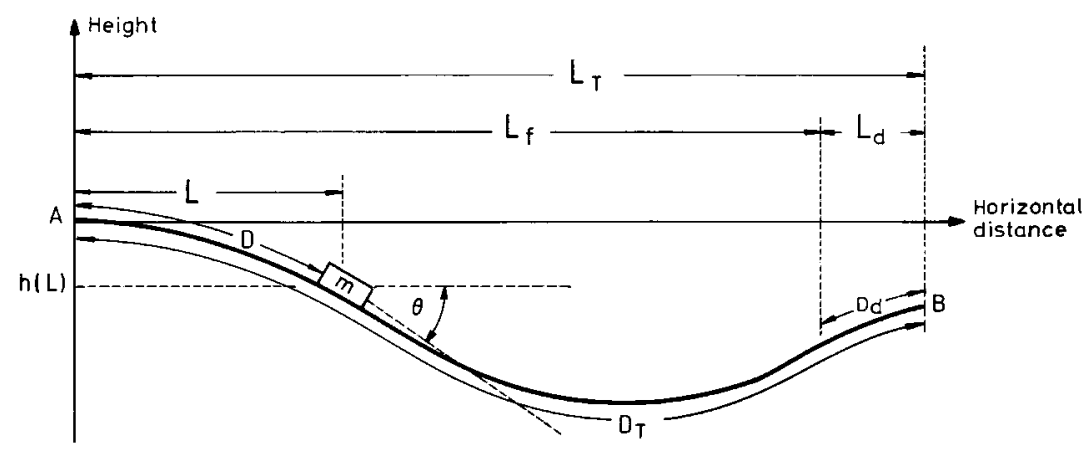

Figure 2. Variable definitions for train model

where $V_{\mathrm{M}}(L)$ denotes the maximum velocity at distance $L$, and $L_{\mathrm{f}}$ is the point at which train starts braking (see Figure 2). In general, this maximum velocity is a function of $L$ to include speed limit zones. In this study we will choose $V_{\mathrm{M}}=16\left(\mathrm{~m} \mathrm{~s}^{-1}\right)$ constant along the whole trajectory.

\subsection{Maximum and minimum acceleration}

From passenger comfort viewpoint, acceleration is not allowed to exceed certain limits either during tractive period or during train braking. These limits depend upon the kind of wheels and type of track. This consideration is taken in to account as

$$
|a(L)| \leqslant A_{\mathrm{M}}, \quad L \in\left[0, L_{\mathrm{T}}\right]
$$

where $A_{\mathrm{M}}$ is the maximum value of acceleration (or deceleration) allowed during braking and traction periods and $L_{\mathrm{T}}$ is the total horizontal distance between two stations (see Figure 2). In our study, since we are not considering the braking period, it can be shown that maximum deceleration due to only gravity and friction effects is $-0.67\left(\mathrm{~m} \mathrm{~s}^{-2}\right)^{12}$ and therefore the constraint is written as $a(L) \leqslant A_{\mathrm{M}} L \in\left[0, L_{\mathrm{f}}\right]$. Taking into consideration that Santiago subway has rubber tires rolling on a special track the value $A_{\mathrm{M}}$ was taken as $1.2\left(\mathrm{~m} \mathrm{~s}^{-2}\right)$.

\subsection{Maximum and minimum tractive force}

As was already stated, propulsion system can only supply a certain maximum power. This fact in turn implies that the tractive force per unit of effective mass generated is constrained as follows:

$$
\Gamma_{\mathrm{m}} \leqslant \gamma(L) \leqslant \Gamma_{\mathrm{M}}, \quad L \in\left[0, L_{\mathrm{f}}\right]
$$

where $\Gamma_{\mathrm{m}} y \Gamma_{\mathrm{M}}$ are the minimum and maximum forces delivered by the propulsion system, respectively.

Since in this case no electric regeneration is being considered $\Gamma_{\mathrm{m}}=0$ and only positive values of $\gamma(L)$ are allowed. From previous studies ${ }^{5}$ it can be shown that this constraint on $\gamma(L)$ is a passive constraint and therefore we will only consider

$$
\gamma(L) \geqslant 0, \quad L \in\left[0, L_{\mathrm{f}}\right]
$$

\subsection{Maximum and minimum track's slope}

The range in which the track slope is allowed to lay is determined by technical considerations such as wheel slip, water drainage and safe parking at stations, amongst others. From this 
consideration we write the constraint

$$
|\theta(L)| \leqslant \theta_{\mathrm{M}}, \quad L \in\left[0, L_{\mathrm{f}}\right]
$$

where $\theta_{\mathrm{M}}$ is the maximum allowed slope. In this work we consider that slope in station vicinity is given by $\theta_{\mathrm{e}}$. Then we can write

$$
\theta_{\mathrm{M}}= \begin{cases}\theta_{\mathrm{e}} & \text { if } 0<L \leqslant L_{\mathrm{e}} \\ \theta_{\mathrm{m}} & \text { if } L_{\mathrm{e}}<L \leqslant L_{\mathrm{f}}\end{cases}
$$

where $L_{\mathrm{e}}$ is chosen as half of the train length, which in the case of Santiago subway is $L_{\mathrm{e}}=70 \mathrm{~m}$ (see Figure 7). In this study $\theta_{\mathrm{e}}=0.002$ and $\theta_{\mathrm{m}}=0.06$ were considered.

Equations (2)-(5) form the set of constraints to be considered in the solution of the problem. This set of constraints can be rewritten as ${ }^{13}$

$$
\begin{gathered}
v(L) V_{\mathrm{M}}-v^{2}(L)>0, \\
{\left[A_{\mathrm{M}}-v(L) \mathrm{d} v / \mathrm{d} L\right] \geqslant 0} \\
\gamma(L) \geqslant 0, \\
{\left[\theta_{\mathrm{M}}+\theta(L)\right]\left[\theta_{\mathrm{M}}-\theta(L)\right) \geqslant 0}
\end{gathered}
$$

\subsection{Boundary conditions}

Besides the previous set of constraints, we shall consider the following boundary and initial conditions:

(1) $t_{0}=0$; initial time.

(2) $t_{\mathrm{f}}=T_{\mathrm{f}}$; final time given from traffic considerations and $T_{\mathrm{f}}=T_{\mathrm{T}}-T_{\mathrm{B}}$, where $T_{\mathrm{T}}$ is the total time between stations and $T_{\mathrm{B}}$ is the braking time (time needed to bring train velocity to zero).

(3) $v_{a}\left(t_{0}\right)=v_{b}\left(t_{0}\right)=0$; initial velocity.

(4) $v_{a}\left(t_{\mathrm{f}}\right)=v_{b}\left(t_{\mathrm{f}}\right)=V_{\mathrm{F}}$; final velocity given from braking considerations. It is assumed that if train reaches $L_{\mathrm{f}}$ at time $t_{\mathrm{f}}$ with speed $V_{\mathrm{F}}$ there exists a suitable braking system to bring train velocity to zero. Since in this study regeneration phenomenon is not considered, braking system is of secondary importance.

(5) $h_{a}(0)=0$; initial height at station A.

(6) $h_{b}(0)=h_{\mathrm{AB}}$, initial height at station $\mathrm{B}$.

(7) $\theta_{a}(L)-\theta_{b}\left(L_{\mathrm{T}}-L\right)=0$ for $L \in\left[L_{\mathrm{d}}, L_{\mathrm{f}}\right]$ where $L_{\mathrm{d}}$ is the braking distance (see Figure 2). This constraint arises since track joining stations $\mathrm{A}$ and $\mathrm{B}$ is unique.

\section{PROBLEM STATEMENT}

In this section we will clearly state the optimization problem including all the constraints mentioned in the above section and the criterion function.

As was previously indicated, our aim is to find an optimal control policy applied to the train as well as an optimal track, such that the energy consumption for a round trip between two consecutive stations is minimized. This problem can be seen as a double optimization problem. Let $E(u, \sigma)$ be the energy consumption for a round trip between two consecutive stations joined by 
a trajectory ' $\sigma$ ' and using a control policy ' $u$ '. The problem is to find $u^{*}$ and $\sigma^{*}$ such that $E\left(u^{*}, \sigma^{*}\right)=E^{*}$ is minimum, i.e.

$$
E^{*}=\min _{u, \sigma} E(u, \sigma)
$$

The performance criterion in this case is defined as the energy consumption for a round trip from stations $\mathrm{A}$ to $\mathrm{B}$ and from stations $\mathrm{B}$ to $\mathrm{A}$. This gives the following criterion function

$$
I=\int_{0}^{L_{\mathrm{f}}}\left\{k_{2}\left[v_{a}^{2}(L)+v_{b}^{2}(L)\right]+k_{3}\left[\theta_{a}(L)+\theta_{b}(L)\right]\right\} \mathrm{d} L
$$

where some constant terms depending upon final and initial conditions have been excluded.

In order to take into account inequality constraints (6) auxiliary control variables $\left(u_{\theta}(L), u_{\mathrm{F}}(L)\right.$, $\left.u_{v}(L), u_{\mathrm{A}}(L)\right)$ and auxiliary state variables $\left(y_{v}(L)\right)$ are introduced $^{13}$ so that the following equality constraints are obtained:

$$
\begin{gathered}
\left.\left[\theta_{\mathrm{M}}+\theta(L)\right]\left[\theta_{\mathrm{M}}-\theta(L)\right)\right]-u_{\theta}^{2}(L)=0 \\
\mathrm{~d} y_{v}(L) / \mathrm{d} L=u_{v}(L) \\
{\left[V_{\mathrm{M}}-2 v(L)\right] \mathrm{d} v(L) / \mathrm{d} L-2 y_{v}(L) u_{v}(L)=0} \\
A_{\mathrm{M}}-v(L) \mathrm{d} v(L) / \mathrm{d} L-u_{\mathrm{A}}^{2}(L)=0 \\
\gamma(L)-u_{\mathrm{F}}^{2}(L)=0
\end{gathered}
$$

The new state variable introduced is $y_{v}(L)$ and the new control variables introduced are $u_{\theta}(L)$, $u_{v}(L), u_{\mathrm{A}}(L)$ and $u_{\mathrm{F}}(L)$. These variables have to be added to the original variables of the problem.

These new equations have to be included twice to consider the case when train is travelling from stations A to B and also when it is travelling from stations B to A.

In summary, the following definition of state and control variables is used in the optimization problem:

$\begin{array}{lcl}\text { State variables } & \text { Control variables } & \\ y_{1}(L)=t_{a} & u_{1}(L)=\gamma_{a} & u_{7}(L)=u_{\mathrm{v} a} \\ y_{2}(L)=t_{b} & u_{2}(L)=\gamma_{b} & u_{8}(L)=u_{\mathrm{v} b} \\ y_{3}(L)=v_{a} & u_{3}(L)=\theta_{a} & u_{9}(L)=u_{\mathrm{A} a} \\ y_{4}(L)=v_{b} & u_{4}(L)=\theta_{b} & u_{10}(L)=u_{\mathrm{A} b} \\ y_{5}(L)=y_{\mathrm{v} a} & u_{5}(L)=u_{\theta a} & u_{11}(L)=u_{\mathrm{F} a} \\ y_{6}(L)=y_{\mathrm{v} b} & u_{6}(L)=u_{\theta b} & u_{12}(L)=u_{\mathrm{F} b}\end{array}$

The state and control vectors are now denoted as $\mathbf{y}(L)=\left[y_{1}(L), \ldots, y_{6}(L)\right]^{\mathrm{T}} \in \mathbb{R}^{6}$ and $\mathbf{u}(L)=\left[u_{1}(L), \ldots, u_{12}(L)\right]^{\mathrm{T}} \in \mathbb{R}^{12}$.

The problem is now stated as to 'Minimize cost function (7) subject to constraints (1) and (8) under initial, final and boundary conditions given in Section 3.5'.

\section{METHOD OF SOLUTION}

In this section a brief general explanation of the numerical method used to solve the problem stated in Section 4 is given. The method chosen to solve the optimization problem stated in the previous section is due to Miele et al. ${ }^{13-16,22}$ and is called gradient-restoration method. This numerical optimization algorithm consists of two clearly distinguishable steps. The first step, called restoration phase $(R)$, takes the so-called nominal functions and applies them to satisfy the 


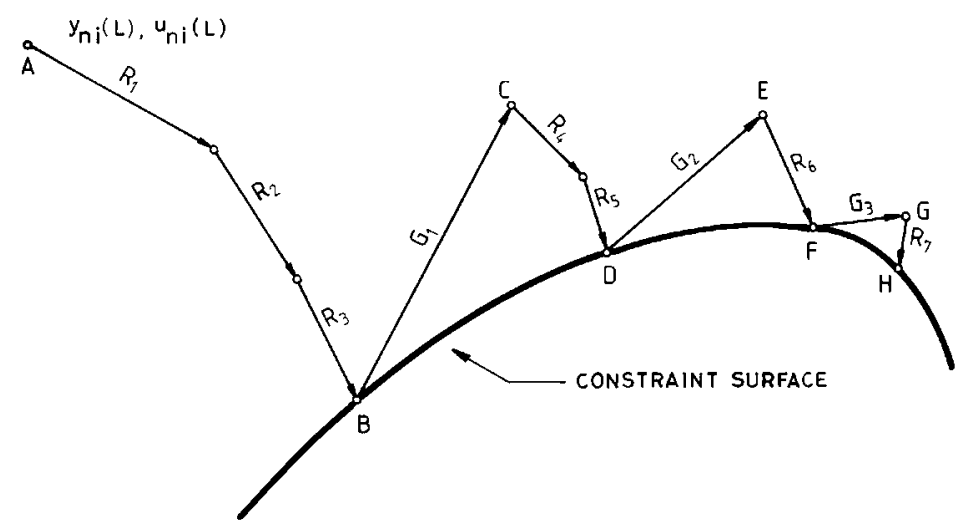

Figure 3. Geometric interpretation of Gradient-Restoration method

problem constraints (equations (1) and (8) in our case). At the beginning (point A in Figure 3), the nominal functions are given $\left(y_{\mathrm{n} i}(L)\right.$ and $u_{\mathrm{n} i}(L)$ in Figure 3$)$ and at any intermediate point these are defined as those from the previous iteration. Restoration phase involves one or more iterations until a scalar performance index $P$, which measures the error in constraints conditions, is sufficiently small (in Figure 3, three iterations are needed to bring the system from A to B and these are denoted by $R_{1}, R_{2}$ and $R_{3}$ ). As soon as this condition is satisfied, the second step, called gradient phase $(G)$, is started. It consists of one iteration and it is designed to decrease the value of the cost function (equation (7) in our case). This phase is characterized by a scalar performance index $Q$ which measures the error in optimality conditions. In Figure 3, first gradient phase brings the system from B to $\mathrm{C}$ and it is denoted by $G_{1}$. The method alternates restoration and gradient phases so that in one of these cycles $(R+G)$ the functional $\mathrm{I}$ is decreased, while problem constraints are satisfied to some pre-specified accuracy. The algorithm is stopped whenever indexes $P$ and $Q$ are simultaneously sufficiently small.

A flow diagram of the gradient restoration method is shown in Figure 4.

This algorithm was implemented in FORTRAN in an IBM 4361 computer. A block diagram of the main program and the corresponding subroutines is shown in Figure 5.

Each subroutine performs the following tasks:

FNOM generates initial nominal functions ${ }^{15,17,18}$

REST performs one restoration phase ${ }^{15,18}$

GRAD performs one gradient phase ${ }^{15,18}$

CALI computes perform index I from (7)

CALQ computes index Q for optimal conditions ${ }^{15}$

CALP computes index $P$ for constraints ${ }^{15}$

LTPBVP solves the linear two point boundary value problem (LTPBVP) generated $^{19-21}$

CALALF computes $\alpha^{*}$ which minimizes augmented functional $\mathbf{J}^{14,15}$

COEF computes coefficients $H_{j k}$ and $F_{j}{ }^{12,15}$

INTERP interpolates the 51 points to get an analytical expression forcoefficients as a function of ' $L$ '12

SET computes a particular solution of LTPBVP 19

EVSFI computes vectors $S$ and $\Phi^{15}$

EVDER computes matrices $S_{y}, S_{u}, \Phi_{y}$ and $\Phi_{u}$ containing partial derivative ${ }^{15}$

FCN defines functions in LTPBVP to be used by subroutine DVERK (IMSL Library). 


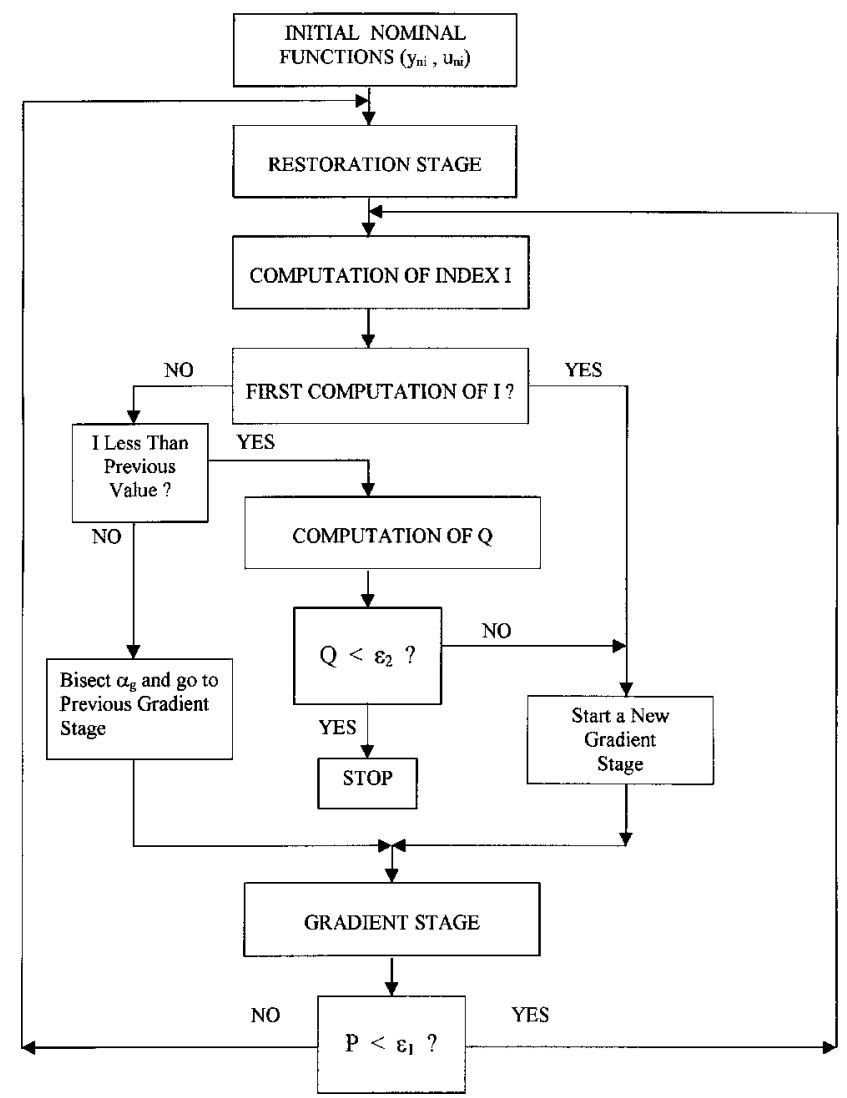

Figure 4. Flow diagram of Gradient-Restoration method

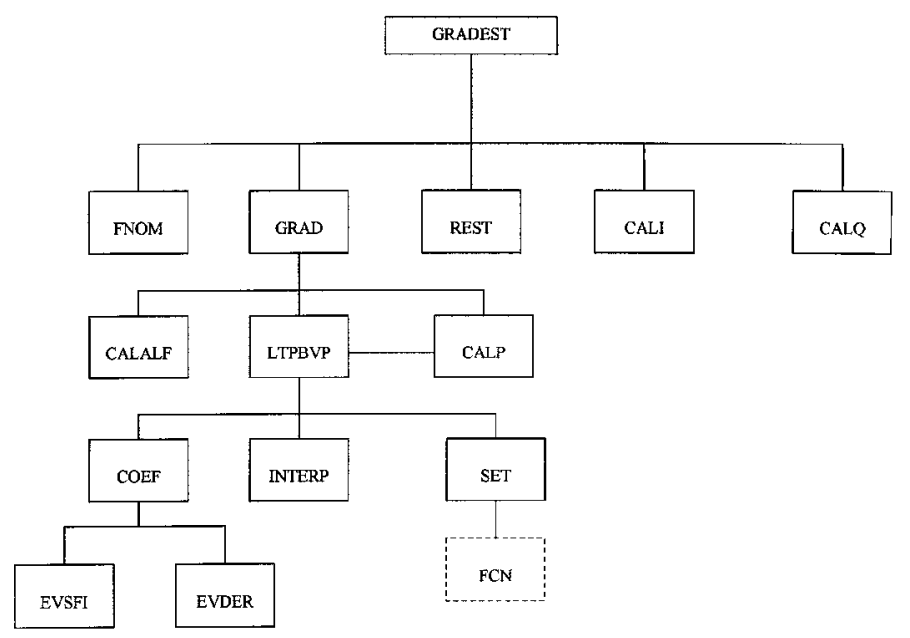

Figure 5. Block diagram of Gradient-Restoration program 
The average execution time of the computational program used in solving one pair of stations was about $10 \mathrm{~min}$.

\section{SIMULATION RESULTS}

The problem stated in Section 4 was solved using the algorithm presented in Section 5. For this simulation the following numerical values of the parameter and constraints values were used

$$
\begin{gathered}
k_{1}=0.1036 \mathrm{~m} \mathrm{~s}^{-2}, \quad A_{\mathrm{M}}=1.2 \mathrm{~m} \mathrm{~s}^{-2}, \quad \theta_{\mathrm{e}}=0.002 \mathrm{rad} \\
k_{2}=1.065 \times 10^{-4} \mathrm{Kg} \mathrm{s}^{2} \mathrm{~m}^{-1} \quad V_{\mathrm{M}}=16 \mathrm{~m} \mathrm{~s}^{-1}, \quad \theta_{\mathrm{m}}=0.06 \mathrm{rad} \\
k_{3}=8.928 \mathrm{~m} \mathrm{~s}^{-2}, \quad L_{\mathrm{e}}=70 \mathrm{~m}, \quad M=1.847 \times 10^{5} \mathrm{Kg} \\
g=9.8 \mathrm{~m} \mathrm{~s}^{-2} \quad L_{\mathrm{d}}=140 \mathrm{~m} \quad m=2.027 \times 10^{5} \mathrm{Kg}
\end{gathered}
$$

These values correspond to the type of rolling material used in the Santiago subway trains. Topology in the equivalent vertical plane corresponding to Stations of Line No. 1 (Escuela Militar-San Pablo) of Santiago subway was chosen in the study. Actual and computationally obtained trajectories were compared.

Five car trains were used in the study whose composition is three tractive, one pilot and one trailer cars. For simulations, the interval of integration was divided into 50 steps and double precision arithmetic was used.

The energy consumption for actual and optimal tracks are shown in Table I. An average energy saving of $18 \%$ per train is reached if a whole round trip is considered. This energy saving is obtained comparing the actual Line No. 1 track profile using an optimal control policy generated by the computer program, versus the computationally obtained ideal track together with an optimal control policy, both generated by the computer program (solution of the double optimization problem.

A typical result between two pairs of station is shown in Figure 6. Velocity and height as functions of horizontal distance ' $L$ ' are shown for both actual and ideal cases.

This case corresponds to La Moneda-Universidad de Chile stations (TR12) and has the following characteristics:

$$
\begin{gathered}
L_{\mathrm{T}}=458 \mathrm{~m}, \quad T_{\mathrm{f}}=35 \mathrm{~s} \\
L_{\mathrm{f}}=318 \mathrm{~m}, \quad h_{\mathrm{BA}}=-1 \cdot 11 \mathrm{~m}
\end{gathered}
$$

From Table I it is possible to distinguish some extreme cases, which are commented in what follows.

(1) Maximum energy consumption: This situation occurs for stations denoted a TR2 (Neptuno-Pajaritos) where energy consumption is $22 \cdot 6$ and $19 \cdot 30 \mathrm{KWH}$ for actual and proposed tunnel profiles, respectively. The distance between stations in this case is the largest of the whole circuit of Line No. $1\left(L_{\mathrm{T}}=990 \mathrm{~m}\right)$ and the ratio $h_{\mathrm{BA}} / L_{\mathrm{T}}$ is the shortest $\left(h_{\mathrm{BA}} / L_{\mathrm{T}}=0 \cdot 00158\right)$.

(2) Minimum energy consumption: This case corresponds to stations denoted as TR12 (La Moneda-Universidad de Chile) and energy consumption are 9.80 and 6.77 KWH for actual and proposed trajectories, respectively. This situation corresponds to the shortest distance between stations in the whole Line No. 1 circuit $\left(L_{\mathrm{T}}=458 \mathrm{~m}\right)$ and it has the less level difference between stations $\left(h_{\mathrm{BA}}=-1.11 \mathrm{~m}\right)$ in the circuit. 
Table I. Energy consumption for Line No. 1 using actual and proposed tunnel profiles

\begin{tabular}{|c|c|c|c|c|c|c|c|c|}
\hline \multirow[t]{2}{*}{ Stations } & \multicolumn{3}{|c|}{ Actual trajectory } & \multicolumn{3}{|c|}{ Optimal trajectory } & \multicolumn{2}{|c|}{ Energy saving } \\
\hline & $\begin{array}{c}\text { Energy } \\
\text { from A to B } \\
\text { (KWH) }\end{array}$ & $\begin{array}{c}\text { Energy } \\
\text { from B to A } \\
(\mathrm{KWH})\end{array}$ & $\begin{array}{l}\text { Total } \\
\text { energy } \\
\text { (KWH) }\end{array}$ & $\begin{array}{c}\text { Energy } \\
\text { from A to B } \\
(\mathrm{KWH})\end{array}$ & $\begin{array}{c}\text { Energy } \\
\text { from B to A } \\
\text { (KWH) }\end{array}$ & $\begin{array}{l}\text { Total } \\
\text { energy } \\
(\mathrm{KWH})\end{array}$ & $(\mathrm{KWH})$ & $(\%)$ \\
\hline TR1 & 6.83 & $10 \cdot 10$ & 16.90 & $5 \cdot 53$ & $8 \cdot 62$ & $14 \cdot 20$ & $2 \cdot 76$ & $16 \cdot 31$ \\
\hline TR2 & $12 \cdot 10$ & $10 \cdot 60$ & $22 \cdot 60$ & $10 \cdot 60$ & $8 \cdot 78$ & $19 \cdot 30$ & $3 \cdot 29$ & $14 \cdot 54$ \\
\hline TR3 & $11 \cdot 60$ & $10 \cdot 40$ & $22 \cdot 00$ & $10 \cdot 10$ & 8.69 & $18 \cdot 80$ & $3 \cdot 24$ & $14 \cdot 71$ \\
\hline TR4 & $9 \cdot 96$ & $7 \cdot 19$ & $17 \cdot 10$ & $8 \cdot 35$ & 5.67 & $14 \cdot 00$ & $3 \cdot 12$ & $18 \cdot 22$ \\
\hline TR5 & $10 \cdot 60$ & 6.71 & $17 \cdot 30$ & $8 \cdot 87$ & $5 \cdot 22$ & $14 \cdot 10$ & $3 \cdot 18$ & $18 \cdot 39$ \\
\hline TR6 & $8 \cdot 66$ & 5.67 & $14 \cdot 30$ & $7 \cdot 32$ & $4 \cdot 45$ & $11 \cdot 80$ & $2 \cdot 56$ & $17 \cdot 87$ \\
\hline TR7 & $9 \cdot 52$ & $7 \cdot 44$ & $17 \cdot 00$ & $8 \cdot 24$ & $5 \cdot 86$ & $14 \cdot 10$ & $2 \cdot 87$ & 16.91 \\
\hline TR8 & $9 \cdot 33$ & 3.93 & $13 \cdot 30$ & $7 \cdot 73$ & $3 \cdot 05$ & $10 \cdot 80$ & $2 \cdot 47$ & 18.66 \\
\hline TR9 & $10 \cdot 70$ & 5.09 & $15 \cdot 80$ & $9 \cdot 08$ & $3 \cdot 80$ & $12 \cdot 90$ & $2 \cdot 94$ & $18 \cdot 60$ \\
\hline TR10 & $8 \cdot 49$ & $5 \cdot 49$ & $14 \cdot 00$ & $7 \cdot 03$ & $4 \cdot 23$ & $11 \cdot 30$ & $2 \cdot 72$ & $19 \cdot 48$ \\
\hline TR11 & $9 \cdot 70$ & $4 \cdot 13$ & $13 \cdot 80$ & $8 \cdot 16$ & $3 \cdot 04$ & $11 \cdot 20$ & $2 \cdot 64$ & $19 \cdot 07$ \\
\hline TR12 & $4 \cdot 29$ & $5 \cdot 51$ & $9 \cdot 80$ & $2 \cdot 85$ & $3 \cdot 91$ & 6.77 & $3 \cdot 04$ & $30 \cdot 97$ \\
\hline TR13 & $11 \cdot 20$ & $2 \cdot 43$ & 13.60 & $9 \cdot 31$ & 1.94 & $11 \cdot 20$ & $2 \cdot 34$ & $17 \cdot 24$ \\
\hline TR14 & $9 \cdot 18$ & 4.97 & $14 \cdot 10$ & $7 \cdot 87$ & $3 \cdot 89$ & $11 \cdot 80$ & $2 \cdot 39$ & 16.89 \\
\hline TR15 & $9 \cdot 41$ & $4 \cdot 72$ & $14 \cdot 10$ & 8.04 & 3.66 & $11 \cdot 70$ & $2 \cdot 43$ & $17 \cdot 22$ \\
\hline TR16 & 16.50 & $5 \cdot 27$ & $21 \cdot 70$ & $14 \cdot 00$ & $4 \cdot 22$ & $18 \cdot 20$ & $3 \cdot 52$ & $16 \cdot 21$ \\
\hline TR17 & $11 \cdot 10$ & $6 \cdot 39$ & $17 \cdot 50$ & $9 \cdot 29$ & $4 \cdot 91$ & $14 \cdot 20$ & $3 \cdot 27$ & $18 \cdot 73$ \\
\hline TR18 & 11.60 & $5 \cdot 54$ & $17 \cdot 10$ & $9 \cdot 66$ & $4 \cdot 26$ & 13.90 & $3 \cdot 20$ & 18.67 \\
\hline TR19 & $10 \cdot 90$ & $4 \cdot 48$ & $15 \cdot 40$ & $9 \cdot 21$ & $3 \cdot 47$ & $12 \cdot 70$ & 2.69 & $17 \cdot 51$ \\
\hline TR20 & $12 \cdot 00$ & $3 \cdot 86$ & $15 \cdot 80$ & $9 \cdot 98$ & $2 \cdot 79$ & $12 \cdot 80$ & $3 \cdot 08$ & $19 \cdot 43$ \\
\hline TR21 & $11 \cdot 50$ & $3 \cdot 71$ & $15 \cdot 20$ & $9 \cdot 72$ & $2 \cdot 91$ & $12 \cdot 60$ & 2.56 & $16 \cdot 84$ \\
\hline TR22 & $10 \cdot 80$ & $4 \cdot 64$ & $15 \cdot 50$ & $9 \cdot 16$ & $3 \cdot 47$ & $12 \cdot 60$ & $2 \cdot 86$ & $18 \cdot 45$ \\
\hline TR23 & $12 \cdot 30$ & $3 \cdot 43$ & $15 \cdot 70$ & $10 \cdot 10$ & $2 \cdot 54$ & $12 \cdot 60$ & $3 \cdot 11$ & $19 \cdot 79$ \\
\hline Total & & & 370 & & & 303 & $66 \cdot 30$ & $\begin{array}{c}17 \cdot 93 \\
\text { (Average) }\end{array}$ \\
\hline
\end{tabular}

(3) Maximum energy saving: This fact is observed for stations denoted as TR16 (BaquedanoSalvador) and energy saving for round trip is $3.52 \mathrm{KWH}$. The stations here are such that level difference is the largest in the whole Line No. $1\left(h_{\mathrm{BA}}=12.6 \mathrm{~m}\right)$.

(4) Minimum energy saving: This particular case arises between stations denoted as TR13 (Universidad de Chile - Santa Lucía) and the energy saving is of $2.34 \mathrm{KWH}$. These stations are characterized by the largest ratio $h_{\mathrm{BA}} / L_{\mathrm{T}}$ of the whole circuit $\left(h_{\mathrm{BA}} / L_{\mathrm{T}}=0.0236\right)$.

(5) Maximum percent energy saving: This situation occurs for the case indicated in (2) where the minimum energy consumption was experimented.

(6) Minimum percent energy saving: This case corresponds to that mentioned in (1) where the maximum energy consumption was observed.

\section{CONCLUSIONS}

A general methodology to simultaneously generate optimal track as well as optimal control policy for electric railways has been proposed in the paper. This methodology can be used to study in a reliable fashion problems of the type presented here, either at the planning stages of a new metro line or optimizing the operation of existing metro lines. The methodology is 


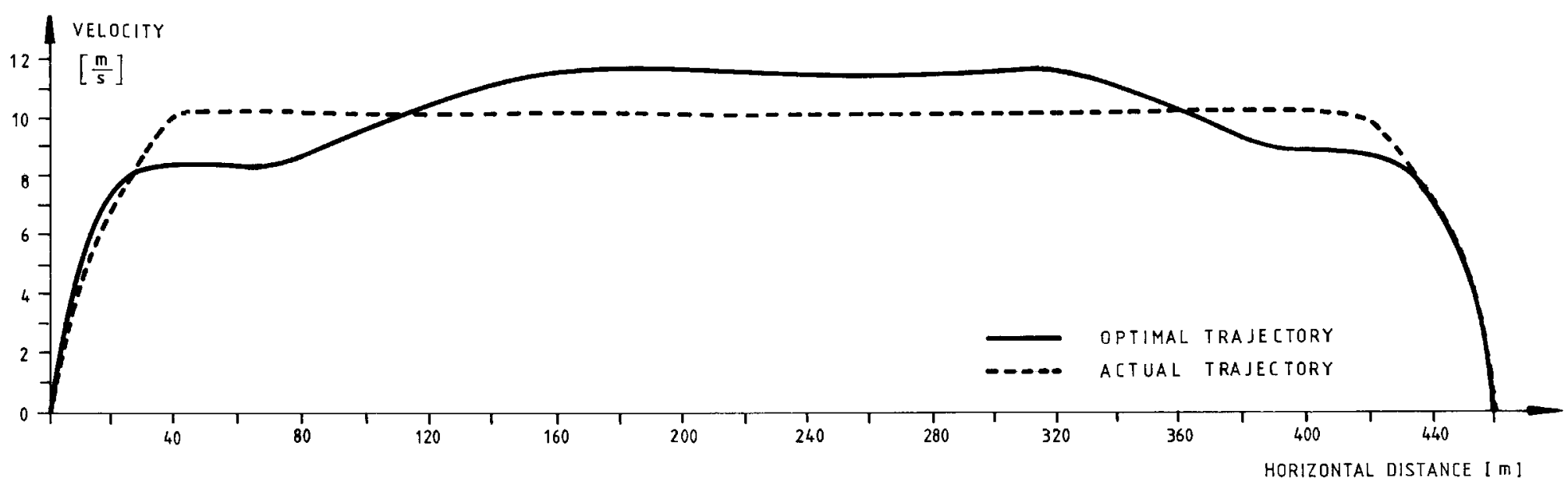

Figure 6. Typical result of optimization method (La Mondeda-U. de Chile). Velocity as a function of distance for actual and proposed trajectories 


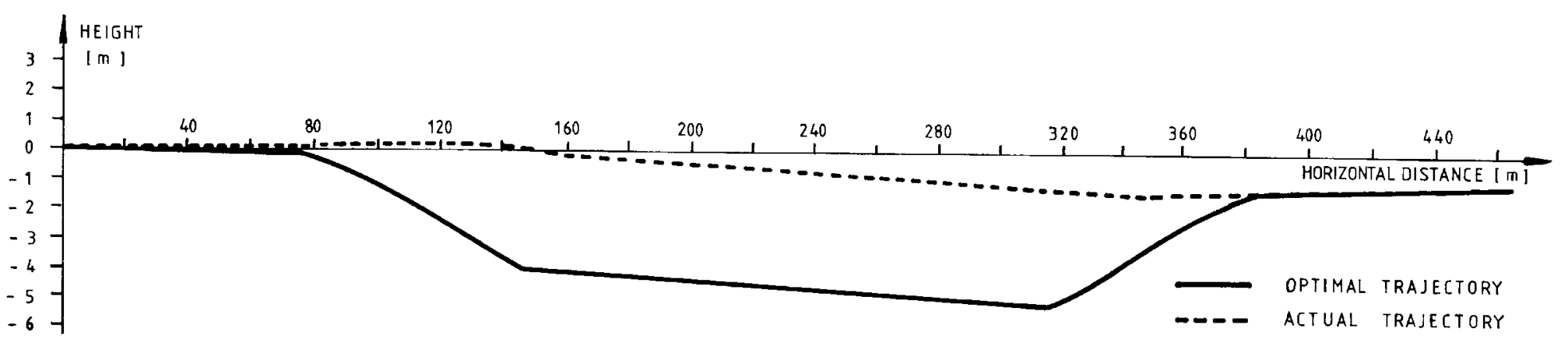

Figure 7. Typical result of optimization method (La Mondeda-U. de Chile). Slope as a function of distance for actual and proposed trajectories 
sufficiently general to include modifications on the train model (e.g. to include electric regeneration during braking periods and other effects) as well as modifications on the numerical method employed to solve the optimization problem, in order to use more proper and efficient numerical methods to solve specific parts of the algorithm.

As a result of applying this methodology to the case of Line No 1 of Santiago subway, an average energy saving of $18 \%$ is obtained for each train traveling a round trip between extreme stations (about $25 \mathrm{~km}$ ). Considering traffic conditions of Line No 1 for years 1989 and 1990, it can be concluded that an energy saving of $4.5 \mathrm{GWh}$ can be achieved yearly (About US\$200.000 per year). From data obtained for later years, these savings are bigger since more trips have been scheduled for the trains and a larger number of trains have been used to satisfy passengers' demands.

In the analysis done in this work, the extra cost of building the optimal track has not been considered in the comparison of actual and proposed trajectories. To make the comparison fair enough, an exhaustive economic analysis which includes this factor should be done before considering the possibility of designing optimal tracks using this methodology.

\section{REFERENCES}

1. Ichikawa, K., 'Application of optimization theory for bounded state variable problems to the operation of train', Bull. JSME, 11(47), 857-865 (1968).

2. Kokotovic, P. and G. Singh, 'Minimum-energy control of a traction motor', IEEE Trans. Automat. Control, 17(1), 92-95 (1972).

3. Silva, J. P., 'Optimal control of traction motors with state transfer', Master of Science Thesis, E.E. Department, University of Chile, Santiago, Chile, 1975 (in Spanish).

4. Talukdar, S. N. and R. L. Koo, "Multiobjective trajectory optimization for electric trains', IEEE Trans. Automat. Control, 24(6), 888-893 (1979).

5. Ortíz, G., 'Optimal control applied to underground railways', Electrical Engineering Thesis, E.E. Department, University of Chile, Santiago, Chile, 1982 (in Spanish).

6. Gruber P. and M. M. Bayoumi, 'Suboptimal control strategies for multilocomotive powered trains', IEEE Trans. Automat. Control, 27(3), 536-546 (1982).

7. Duarte, M. A., 'General considerations about minimum energy consumption of underground railways', Proc. I Chilean Conference on Transportation Engineering. Santiago, Chile, 1984, pp. 308-320 (in Spanish).

8. Mattis, W. E., 'Modelling and minimum energy control of traction motor systems', Int. J. Energy Systems, 9(2), 78-82 (1989).

9. Duarte, M. A. and G. Ortíz, 'Indirect adaptive control of underground railways', Proc. IV Brazilian Conf. on Automatic Control, Campinas, Brazil, Vol. 1, 1982, pp. 137-142 (in Spanish).

10. Hoang, H. H., M. P. Polis and A. Haurie, 'Reducing energy consumption through trajectory optimization for a metro network', IEEE Trans. Automat. Control, 20(5), 590-595 (1975).

11. Duarte, M. A. and N. Haddad, 'On the determination of minimum energy trajectories for an underground railway network', Proc. V Chilean Conf. on Automatic Control, Valparaíso, Chile. Vol. I, 1982, pp. 280-299 (in Spanish).

12. Sotomayor, P., 'On the determination of optimal trajectories for underground railways', Electrical Engineering Thesis, E.E. Department, University of Chile, Santiago, Chile, 1990 (in Spanish).

13. Miele, A., 'Recent advances in gradient algorithms for optimal control problems', J. Optim. Theory Appl., 17(5/6), 361-430 (1975).

14. Miele, A., J. N. Damoulakis, J. R. Cloutier and J. L. Tietze, 'Sequential gradient-restoration algorithm for optimal control problems with nondifferential constraints', J. Optim. Theory Appl., 13(2), 218-255 (1974).

15. Miele, A., 'Gradient algorithms for the optimization of dynamic systems', in Leondes, C. T. (ed.), Control and Dynamic Systems: Advances in Theory and Applications, Vol. 16, Academic Press, New York, 1980, pp. $1-52$.

16. Miele, A. and T. Wang, 'Supplementary optimality properties of the restoration phase of sequential gradient-restoration algorithms for optimal control problems', J. Optim. Theory Appl., 41(1), 169-184 (1983). 
17. Miele, A. and J. N. Damoulakis, 'Modifications and extensions of the sequential gradient-restoration algorithm for optimal control theory', J. Franklin Inst., 294(1), 23-42 (1972).

18. González, S. and A. Miele, 'Sequential gradient-restoration algorithm for optimal control problems with general boundary conditions', J. Optim. Theory Appl., 26(3), 395-425 (1978).

19. Miele, A., 'Method of particular solutions for linear two-point boundary-value problems', J. Optim. Theory Appl., 2(4), 260-273 (1968).

20. Miele, A. and R. R. Iyer, 'General technique for solving nonlinear, two-point boundary-value problems, via the method of particular solutions', J. Optim. Theory Appl., 5(5), 382-399 (1970).

21. Miele, A. and R. R. Iyer, 'Modified quasilinearization method for solving nonlinear, two-point boundary-value problems', J. Math. Anal. Appl., 36(3), 247-256 (1971).

22. Henning G. R. and A. Miele, 'Sequential gradient-restoration algorithm for optimal control problems with bounded state', J. Optim. Theory Appl., 12(1), 61-98 (1973). 